\title{
Análise dos Motivos de Insatisfatoriedade dos Exames Histopatológicos do Colo do Útero no Sistema Único de Saúde, Brasil, 2014 a 2017
}

doi: https://doi.org/10.32635/2176-9745.RBC.2021v67n3.1299

\author{
Analysis of The Reasons for Unsatisfactory Cervical Histopathological Exams in the Unified Health System, Brazil, 2014 to 2017 \\ Análisis de las Causas de los Exámenes Histopatológicos Cervicales Insatisfactorios en el Sistema Único de Salud, Brasil, \\ 2014 a 2017
}

Itamar Bento Claro'; Mário Lúcio Cordeiro Araújo Junior²; Arn Migowski³; Jeane Glaucia Tomazelli

\section{RESUMO}

Introduçáo: $\mathrm{O}$ câncer do colo do útero é ainda um importante problema de saúde pública no Brasil e no mundo. A qualidade dos exames de rastreio e de confirmação diagnóstica são fundamentais para que as açôes de controle do câncer alcancem efetivo impacto sobre a mortalidade. Objetivo: Analisar as informaçóes registradas no campo descritivo dos motivos da insatisfatoriedade dos exames histopatológicos do colo do útero. Método: Estudo descritivo com dados do Sistema de Informação do Câncer (SISCAN) de mulheres que realizaram exame histopatológico do colo do útero no Sistema Único de Saúde (SUS) no período de 2014 a 2017. Foram analisados 1.236 exames histopatológicos do colo do útero. Os motivos de insatisfatoriedade especificados no campo descritivo foram analisados, reclassificados e, quando pertinente, foram redistribuídos nos campos existentes para exames satisfatórios. Resultados: Foram classificados incorretamente como 'insatisfatório' 262 exames (21,2\%), dos quais, 11,25\% foram reclassificados como lesão de caráter benigno, 5,91\% como lesão de alto grau, $1,46 \%$ como carcinoma, 0,24\% como adenocarcinoma e 0,24\% como adenocarcinoma in situ. Conclusáo: $\mathrm{O}$ estudo demonstrou um percentual expressivo de erros nos laudos classificados como insatisfatórios no SUS, apontando a necessidade de capacitar profissionais que emitem laudo histopatológico de forma a evitar erros diagnósticos.

Palavras-chave: Neoplasias do Colo do Útero; Sistema Único de Saúde; Teste de Papanicolaou; Programas de Rastreamento; Patologia Clínica.

\section{ABSTRACT}

Introduction: Cervical cancer is still an important public health problem in Brazil and worldwide. The quality of screening and diagnostic confirmation tests are essential for cancer control actions to achieve an effective impact on mortality. Objective: The aim of this study was to analyze the information registered in the description field of the motives for the unsatisfactoriness of the histopathological exams of the cervix. Method: Descriptive study using data of the Cancer Information System (SISCAN) of women who underwent histopathological examination of the cervix in the Unified Health System (SUS) in the period from 2014 to 2017. 1,236 histopathological examinations of the cervix were analyzed. The reasons for unsatisfactoriness specified in the description field were analyzed and reclassified and, when relevant, were reassigned to the existing fields for satisfactory exams. Results: 262 exams (21.2\%) were incorrectly classified as 'unsatisfactory', in which $11.25 \%$ were reclassified as a benign lesion, $5.91 \%$ as a highgrade lesion, $1.46 \%$ as carcinoma, $0.24 \%$ as adenocarcinoma and $0.24 \%$ as adenocarcinoma in situ. Conclusion: The study showed a significant percentage of errors in reports classified as unsatisfactory in SUS, pointing out the need to train professionals who issue histopathological reports in order to avoid diagnostic errors.

Key words: Uterine Cervical Neoplasms; Unified Health System; Teste de Papanicolaou; Mass Screening; Pathology, Clinical.

\section{RESUMEN}

Introducción: El cáncer de cuello uterino sigue siendo un importante problema de salud pública en Brasil y en todo el mundo. La calidad de las pruebas de cribado y las de confirmación diagnóstica son fundamentales para que las acciones de control del cáncer logren un impacto efectivo en la mortalidad. Objetivo: El objetivo de este estudio fue analizar la información registrada en el campo descriptivo de las razones de la insatisfacción de los exámenes histopatológicos del cuello uterino. Método: Estudio descriptivo que utilizó datos del Sistema de Información del Cáncer (SISCAN) de mujeres a las que se les realizó examen histopatológico cervicouterino en el Sistema Único de Salud (SUS) de 2014 a 2017. Se analizaron 1.236 exámenes histopatológicos cervicouterino. útero. Los motivos de insatisfacción especificados en el campo descriptivo se analizaron y reclasificaron y, cuando fue relevante, se redistribuyeron en los campos existentes de satisfacción con el examen. Resultados: 262 exámenes $(21,2 \%)$ se clasificaron incorrectamente como 'insatisfactorios', en los que el 11,25\% se reclasificó como lesión benigna, el 5,91\% como lesión de alto grado, el $1,46 \%$ como carcinoma, $0,24 \%$ como adenocarcinoma y $0,24 \%$ como adenocarcinoma in situ. Conclusión: El estudio mostró un porcentaje significativo de errores en informes calificados como insatisfactorios en el SUS, señalando la necesidad de formar profesionales que emitan informes histopatológicos para evitar errores diagnósticos.

Palabras clave: Neoplasias del Cuello Uterino; Sistema Único de Salud; Prueba de Papanicolaou; Tamizaje Masivo; Patología Clínica.

\footnotetext{
${ }^{1-4}$ Instituto Nacional de Câncer José Alencar Gomes da Silva (INCA). Rio de Janeiro (RJ), Brasil.

'E-mail: iclaro@hotmail.com. Orcid iD: https://orcid.org/0000-0002-6435-3302

2E-mail: mljunior@inca.gov.br. Orcid iD: https://orcid.org/0000-0002-3823-7690

3E-mail: arnmigowski@yahoo.com.br. Orcid iD: https://orcid.org/0000-0002-4861-2319

${ }^{4}$ E-mail: jtomazelli@inca.gov.br. Orcid iD: https://orcid.org/0000-0002-2472-3444

Endereço para correspondência: Itamar Bento Claro. Rua Marquês de Pombal, 125, $7^{\circ}$ andar - Centro. Rio de Janeiro (RJ), Brasil. CEP 20230-240.
} 


\section{INTRODUÇÃO}

O câncer do colo do útero é ainda um importante problema de saúde pública no mundo. Sua incidência e mortalidade são maiores nos países de menor renda ${ }^{1}$. Neles, predominam açóes de rastreamento caracterizadas como oportunísticas, nas quais o rastreio é ofertado quando as mulheres acessam os serviços de saúde por outros motivos ${ }^{2}$. Portanto, não há um programa de rastreamento, com idade e periodicidade definidas, no qual as mulheres sejam formalmente convidadas a participar. Esse modelo oportunístico é menos efetivo no impacto sobre a morbimortalidade e costuma ser mais oneroso para o sistema de saúde ${ }^{3,4}$.

O Brasil ainda apresenta elevada incidência e mortalidade por câncer do colo do útero, com um risco estimado de 12,6 por 100 mil mulheres, em 2020, correspondendo a 16.590 casos novos, sendo a terceira neoplasia mais frequente, excluíndo o câncer de pele não melanoma ${ }^{5}$. Apesar de ser um câncer altamente curável, em 2018 foram registrados 6.526 óbitos por essa neoplasia no país ${ }^{6}$. Verifica-se uma persistência de estabilidade nas taxas de mortalidade ao longo das últimas décadas, apesar da queda em capitais 7 . Porém, estudo recente sobre tendência de mortalidade por câncer indica queda da taxa de mortalidade por câncer do colo do útero em todo país, exceto no interior da Regiáo Norte ${ }^{8}$, o que leva a refletir se as açôes de controle estão sendo conduzidas de forma adequada. A redução da incidência e mortalidade são alcançadas com as açôes efetivas de prevenção primária (vacina contra HPV), detecção precoce e tratamento das lesôes precursoras e encaminhamento oportuno para tratamento dos casos de câncer diagnosticados? .

A qualidade diagnóstica dos exames citopatológicos, técnica de rastreio adotada no Brasil, e histopatológicos do colo do útero são fundamentais para que as açóes de controle do câncer alcancem o sucesso esperado. Para tanto, se faz necessário reduzir o percentual de exames falso-negativos, falso-positivos e insatisfatórios. A qualificação profissional para o preparo da lâmina é condição sine qua non, pois além da realização de uma boa coleta, o esfregaço precisa ser feito de forma bem distribuída e adequadamente fixado para melhor visualização quando a lâmina for corada. $\mathrm{O}$ ideal para a realização do exame citopatológico é que o esfregaço contenha a representação dos epitélios escamoso, glandular e/ou metaplásico. Contudo, para ser considerado satisfatório para análise, poderá conter apenas células do epitélio escamoso ${ }^{10,11}$.

As atividades de controle e garantia da qualidade do exame citológico assumem vital importância nas açôes de rastreamento e as correlaçôes cito-histológica e clínica fazem parte das diferentes estratégias utilizadas ${ }^{11,12}$. $\mathrm{O}$ exame histopatológico se baseia em critérios morfológicos arquitetural e celular, ele representa o padrão-ouro do diagnóstico morfológico ${ }^{13}$.

O exame histopatológico é necessário para excluir erros de diagnóstico e estabelecer a conduta adequada ${ }^{14}$ e constitui o principal instrumento para o diagnóstico de confirmação de uma neoplasia pré-maligna ou maligna. É realizado a partir de uma amostra de tecido, obtida por meio de biópsia, após o encaminhamento da mulher à colposcopia. Possibilita o diagnóstico de lesôes náo visualizadas na colposcopia e, algumas vezes, pode apresentar um diagnóstico diferente do exame citopatológico; por isso, é importante que os resultados histopatológicos sejam expressos segundo uma nomenclatura que permita a correlação cito-histológica. $\mathrm{Na}$ análise microscópica, é feita a identificação da natureza da lesão, particularizando-se as lesões de caráter benigno e as de caráter pré-neoplásico ou neoplásico, definindo, sempre que possível, o grau de diferenciação, a extensão do tumor e as margens cirúrgicas ${ }^{15,16}$.

O resultado do exame histopatológico norteia as decisóes de tratamento e seguimento clínico e, por isso, a qualidade de todo o processo diagnóstico precisa ser garantida e reduzida as dificuldades para melhorar a acurácia do exame. Fatores como subjetividade e experiência do examinador, orientação e espessura do corte podem influenciar a interpretação do exame.

O objetivo deste estudo foi analisar as informaçóes descritas no campo dos motivos da insatisfatoriedade dos exames histopatológicos do colo do útero registrados no Sistema de Informação do Câncer (SISCAN), no período de 2014 a 2017, no Brasil.

\section{MÉTODO}

Trata-se de um estudo transversal descritivo sobre as causas de insatisfatoriedade relacionadas aos exames histopatológicos do colo do útero registrados no SISCAN no período de 2014 a 2017.

Os resultados dos exames histopatológicos do colo do útero são registrados em um formulário padronizado nacionalmente para captação dos dados a serem informados no SISCAN. Nesse formulário, não há as especificaçôes pré-selecionadas para insatisfatoriedade do material como ocorre no exame citopatológico ${ }^{16}$. Para registro dos exames citopatológicos, quando o material é considerado insatisfatório para avaliação oncótica, é possível selecionar, entre os campos disponíveis, o motivo da não adequabilidade: material acelular ou hipocelular em menos de 10\% do esfregaço; sangue em mais de $75 \%$ do esfregaço; piócitos em mais de $75 \%$ do 
esfregaço; artefatos de dessecamento em mais de $75 \%$ do esfregaço; contaminantes externos em mais de $75 \%$ do esfregaço; intensa superposição celular em mais de 75\% do esfregaço e ainda especificar outras possíveis causas não relacionadas ${ }^{17}$.

No campo denominado resultado do exame histopatológico, o médico anatomopatologista precisa especificar o tipo de procedimento cirúrgico que originou a amostra, quais sejam: biópsia, exérese da zona de transformação, conização, histerectomia simples, histerectomia com anexectomia unilateral ou bilateral ou classificar como outros e discriminar por escrito (Figura 1).

No resultado da microscopia (Figura 2), deverá ser informado o tipo de material recebido, se biópsia ou peça cirúrgica; a localização do tumor (ectovérvice, endocérvice ou junção escamocolunar) e a adequabilidade do material (satisfatório ou insatisfatório). Se o material for satisfatório, seguirá a análise da microspia na qual será especificado o tipo de lesão de caráter benigno, neoplásico ou pré-neoplásico. No caso de o material ser considerado insatisfatório, o profissional deverá especificar em campo aberto o motivo da insatisfatoriedade, uma vez que não há especificações pré-categorizadas no formulário desse exame.

Neste trabalho, foram analisados os motivos de insatisfatoriedade especificados no campo descritivo. Os motivos descritos foram categorizados e, nos casos em que foi detectado erro do uso do campo livre, com descrição de diagnósticos em vez de motivos insatisfatoriedade, estes foram reclassificados e, quando pertinente, redistribuídos nos campos existentes de satisfatoriedade do exame com descrição compatível. A pesquisa ocorreu no ano de 2018 e foram utilizados todos os registros histopatológicos informados no SISCAN nesse período, sendo o mesmo definido considerando a disponibilidade da base de dados consolidada e o ano de início do estudo.

Os registros histopatológicos com informação de insatisfatoriedade foram extraídos da base de dados do SISCAN utilizando o programa R, versão 3.5.3, pacote tidyverse $^{18}$, e organizados em planilha Excel, na qual procedeu-se à reclassificação.

Este estudo foi aprovado pelo Comitê de Ética em Pesquisa do Instituto Nacional de Câncer José Alencar Gomes da Silva (INCA), CAAE: 26944219.5.0000.5274.

\section{RESULTADOS}

No período do estudo, havia 137.893 exames histopatológicos do colo do útero registrados no SISCAN, sendo $0,89 \%$ com adequabilidade insatisfatória. Foram analisadas 1.236 descrições no campo de "Insatisfatórios" de todos os exames registrados no SISCAN no período analisado. Após a correçấo ortográfica, permaneceram 882 descrições distintas de insatisfatoriedade.

Verificou-se a utilização de muitos sinônimos para se referir a uma mesma causa de insatisfatoriedade, como, por exemplo, 30 referências para dimensionar como motivo de insatisfatoriedade o tamanho da amostra (Quadro 1). Entre estas, as mais comuns foram: amostra exígua (39), material escasso (28), material insuficiente (22) e amostra escassa (15).

\section{RESULTADO DO EXANE HISTOPATOLOGICO - COLO DO ÚTERO}

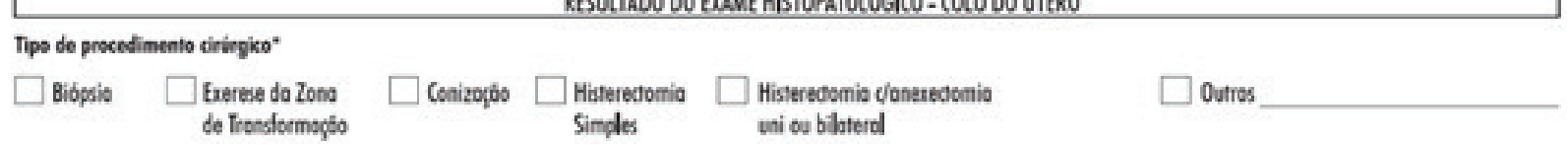

Figura 1. Formulário de requisição de exame histopatológico do colo do útero. Tipo de procedimento cirúrgico Fonte: INCA.

\section{MACROSCOPLA}

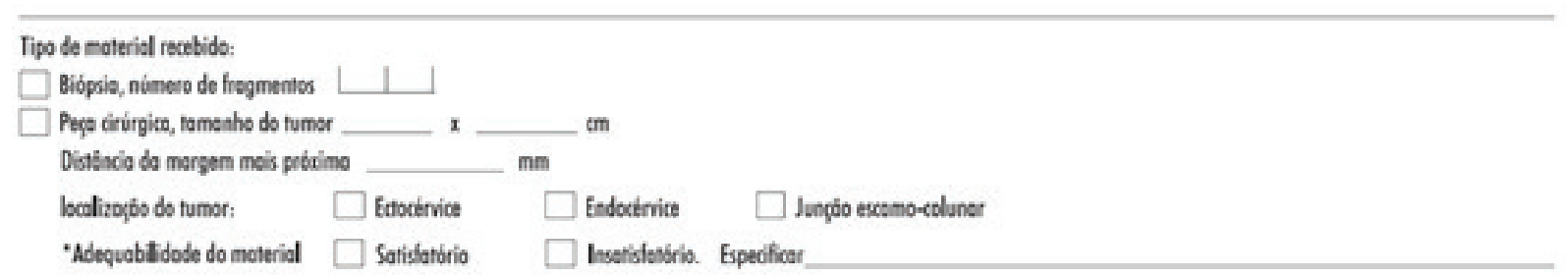

Figura 2. Formulário de requisição de exame histopatológico do colo do útero. Macroscopia Fonte: INCA. 
Quadro 1. Classificação da dimensão do tamanho da amostra no exame histopatológico do colo do útero. Brasil, 2014-2017

\begin{tabular}{|c|c|}
\hline CLASSIFICAÇÃO & ESPECIFICAÇÃO \\
\hline \multirow{7}{*}{ 1. Amostra } & $\begin{array}{l}\text { - de tamanho muito } \\
\text { reduzido }\end{array}$ \\
\hline & - diminuta \\
\hline & - escassa \\
\hline & - exígua \\
\hline & - insuficiente \\
\hline & - muito escassa \\
\hline & - pequena \\
\hline $\begin{array}{l}\text { 2. Biópsia muito } \\
\text { pequena }\end{array}$ & - \\
\hline 3. Diminuto fragmento & - \\
\hline \multirow{4}{*}{ 4. Escassez } & - da amostra \\
\hline & - de elementos \\
\hline & - de tecido \\
\hline & - do material \\
\hline 5. Espécime exígua & - \\
\hline \multirow{3}{*}{ 6. Exiguidade } & - da amostra \\
\hline & - do material \\
\hline & - fragmento \\
\hline \multirow{4}{*}{ 7. Fragmento } & - escasso \\
\hline & - exíguo \\
\hline & - minúsculo \\
\hline & - não representativo \\
\hline \multirow{7}{*}{ 8. Material } & - diminuto \\
\hline & - escasso \\
\hline & - exíguo \\
\hline & - muito exíguo \\
\hline & - muito pequeno \\
\hline & - não representativo \\
\hline & - Pequeno \\
\hline 9. Muito exíguo & - \\
\hline 10. Tamanho reduzido & - \\
\hline
\end{tabular}

Fonte: SISCAN

Verificou-se que 396 exames (32\%) foram classificados incorretamente como 'insatisfatório' com relação à adequabilidade do material. Entre estes, 11,25\% foram reclassificados como lesão de caráter benigno, 5,91\% como lesão de alto grau, 1,46\% como carcinoma, $0,24 \%$ como adenocarcinoma e $0,24 \%$ como adenocarcinoma in situ, tendo sido possível distinguir 22 categorias (Tabela 1).
Tabela 1. Diagnósticos após serem reclassificados. Brasil, 2014-2017

\begin{tabular}{|c|c|c|}
\hline CATEGORIAS & QUANTIDADE & $\%$ \\
\hline Adenocarcinoma & 3 & 0,24 \\
\hline Adenocarcinoma in situ & 3 & 0,24 \\
\hline $\begin{array}{l}\text { Amostra insatisfatória } \\
\text { sem justificativa }\end{array}$ & 34 & 2,75 \\
\hline $\begin{array}{l}\text { Amostra insatisfatória/ } \\
\text { acelular }\end{array}$ & 218 & 17,64 \\
\hline $\begin{array}{l}\text { Amostra insatisfatória/ } \\
\text { ausência de epitélio }\end{array}$ & 112 & 9,06 \\
\hline $\begin{array}{l}\text { Amostra insatisfatória/ } \\
\text { com atipia }\end{array}$ & 14 & 1,13 \\
\hline $\begin{array}{l}\text { Amostra insatisfatória/ } \\
\text { fixação }\end{array}$ & 30 & 2,43 \\
\hline $\begin{array}{l}\text { Amostra insatisfatória/ } \\
\text { fulguração }\end{array}$ & 37 & 2,99 \\
\hline $\begin{array}{l}\text { Amostra insatisfatória/ } \\
\text { hemorrágico }\end{array}$ & 31 & 2,51 \\
\hline $\begin{array}{l}\text { Amostra insatisfatória/ } \\
\text { laboratório }\end{array}$ & 11 & 0,89 \\
\hline $\begin{array}{l}\text { Amostra insatisfatória/ } \\
\text { material escasso }\end{array}$ & 266 & 21,52 \\
\hline $\begin{array}{l}\text { Ausência de um dos } \\
\text { epitélios da JEC }\end{array}$ & 76 & 6,15 \\
\hline Carcinoma & 18 & 1,46 \\
\hline Erro pré-analítico & 27 & 2,18 \\
\hline $\begin{array}{l}\text { Exames de outras áreas } \\
\text { não colo do útero }\end{array}$ & 58 & 4,69 \\
\hline Exames inconclusivos & 60 & 4,85 \\
\hline Lesão de caráter benigno & 139 & 11,25 \\
\hline NIC I & 14 & 1,13 \\
\hline NIC II/III & 73 & 5,91 \\
\hline NIC não graduado & 3 & 0,24 \\
\hline $\begin{array}{l}\text { Outras neoplasias } \\
\text { malignas }\end{array}$ & 2 & 0,16 \\
\hline Sugestivo NIC & 7 & 0,58 \\
\hline Total & 1.236 & 100,00 \\
\hline
\end{tabular}

Fonte: SISCAN.

Legendas: JEC: Junçăo escamocolunar; NIC: Neoplasia intraepitelial cervical.

\section{DISCUSSÃO}

O exame histopatológico tem papel fundamental no diagnóstico do câncer do colo do útero e a realização da imuno-histoquímica pode ser útil para distinguir entre condiçôes benignas e malignas do colo do útero e para discriminar entre os seus vários subtipos ${ }^{19}$.

A identificação de exame histopatológico insatisfatório se dá quando o examinador, ao realizar a análise 
microscópica de um tecido para a detecção de possíveis alterações ou lesóes, não consegue especificar a natureza, a gravidade ou a extensão das alteraçóes na amostra, impossibilitando a conclusão diagnóstica que subsidiaria a definição do tratamento adequado da lesão ${ }^{11}$.

Os resultados encontrados chamaram atenção não apenas pela variabilidade das descriçóes macroscópica evidenciando uma ausência de padrão apropriado, mas principalmente pelo percentual expressivo de exames com resultados equivocadamente classificados como insatisfatórios, muitas vezes apresentando descriçôes de lesão de alto grau ou mesmo câncer que exigiriam encaminhamento imediato para a unidade de referência para realização de tratamento adequado ${ }^{13}$. Não foi encontrada na literatura análise semelhante que possa servir de comparação para os dados do presente estudo. Todavia, estudo com dados dos exames histopatológicos de mama no Sistema Único de Saúde (SUS) indicam que número expressivo de exames histopatológicos de câncer de mama foram classificados como 'outros' em vez de ter seu tipo histológico descrito, o que também remete a problemas de qualidade de exames histopatológico ou de registro da informaçáo no sistema ${ }^{20}$.

A histopatologia fornece o diagnóstico final e serve como padráo-ouro para o controle de qualidade da citologia e da colposcopia ${ }^{21}$. O exame histopatológico, juntamente com os achados clínicos, é obrigatório para o diagnóstico precoce e preciso, a fim de possibilitar o tratamento oportuno ${ }^{22}$. Entretanto, a precisão do diagnóstico histopatológico das amostras de tecido depende de amostras adequadas, descriçáo macroscópica apropriada, processamento técnico, interpretação microscópica e controle de qualidade correlacionando o diagnóstico citológico e histológico ${ }^{19}$. Além da falta de um padrão para especificar a dimensão da amostra, o presente estudo evidencia que são necessárias açôes para aperfeiçoar o controle de qualidade dos exames e treinamento dos profissionais responsáveis pelo laudo para que exames satisfatórios não sejam classificados como insatisfatórios incorretamente, comprometendo a continuidade do cuidado às usuárias.

Os problemas identificados nos laudos insatisfatórios no presente artigo comprometem o cuidado à mulher, sobretudo em uma neoplasia que apresenta ainda elevada magnitude de ocorrência no nosso país. Exames insatisfatórios necessitam de nova coleta de material, o que aumenta a probabilidade de perda de acompanhamento dessas mulheres. Estudos apontam problemas no seguimento da mulher que váo desde o retorno para pegar o laudo do exame citopatológico e a realização de novo exame, se necessário, até a adesão à continuidade das açôes de investigaçáo diagnóstica (colposcopia e histopatológico) ${ }^{23-25}$. Não foi identificado estudo específico que aborde a repetição do exame histopatológico nos casos de insatisfatoriedade, porém todos os fatores envolvidos na linha de cuidado desse câncer, que demandem a necessidade de nova repetição de exame por insatisfatoriedade, devem ser minimizados para garantir maior adesão às condutas necessárias, evitar atrasos diagnósticos e riscos de procedimentos desnecessários.

Os dados são referentes aos serviços que utilizam o SISCAN, portanto não representam a totalidade de exames de todos os prestadores de serviço do SUS. Dados recentes que mostram o grau de implantação SISCAN no país apontam que, em 2017, 74\% dos laboratórios informaram exames histopatológicos do colo do útero nesse sistema ${ }^{4}$. Uma análise por estados ou prestador de serviço não fizeram parte do escopo do trabalho, mas podem contribuir para açôes focais.

\section{CONCLUSÃO}

É de grande importância que os padróes histopatológicos sejam monitorados e laudados em terminologia internacionalmente acordada, pois os resultados da análise histopatológica constituem fonte dos dados de diagnóstico armazenados nos registros de câncer que são usados para avaliação dos programas de rastreamento.

Embora a padronizaçáo do campo de motivos de insatisfatoriedade possa diminuir parte do problema, é necessário compreender o porquê de o profissional avaliar o exame como insatisfatório com relação à adequabilidade do material e simultaneamente colocar nas especificaçóes diagnósticos existentes no campo destinado à microscopia referente às lesôes. Sobretudo porque a não marcação das lesôes de caráter neoplásico ou pré-neoplásico poderá impossibilitar o diagnóstico oportuno, levando ao atraso no tratamento ou até mesmo à perda do seguimento da mulher.

Foram observados problemas na descrição dos exames histopatológicos insatisfatórios e registro de diagnósticos de neoplasias do câncer do colo do útero nesse campo. Esses diagnósticos não são computados nacionalmente dentro dos exames satisfatórios e essas mulheres desconhecem o real diagnóstico. O estudo aponta a necessidade urgente de capacitação dos profissionais que emitem o laudo histopatológico de forma a evitar a perda de seguimento da mulher com exame citopatológico alterado.

\section{CONTRIBUIÇÕES}

Itamar Bento Claro, Mário Lúcio Cordeiro Araújo Junior e Jeane Glaucia Tomazelli contribuíram 
substancialmente na concepção e no planejamento do estudo; na análise e interpretação dos dados, assim como na redação e revisão crítica com contribuição intelectual. Arn Migowski contribuiu na interpretação dos dados da pesquisa e na revisão crítica com contribuição intelectual. Todos os autores aprovaram a versão final a ser publicada.

\section{DECLARAÇÃO DE CONFLITO DE INTERESSES}

Nada a declarar.

\section{FONTES DE FINANCIAMENTO}

Não há.

\section{REFERÊNCIAS}

1. Hull R, Mbele M, Makhafola T, et al. Cervical cancer in low and middle-income countries. Oncol Lett. 2020;20(3):2058-74. doi: https://doi.org/10.3892/ ol.2020.11754

2. International Agency for Research on Cancer. Handbooks of cancer prevention: preamble for secondary prevention. Lyon: International Agency for Research on Cancer; 2019 [cited 2021 June 17]. Available from: https://handbooks. iarc.fr/docs/HB-Preamble-Secondary-Prevention.pdf

3. Maia MN, Silva RPO, Santos LPR. A organização do rastreamento do câncer do colo uterino por uma equipe de Saúde da Família no Rio de Janeiro, Brasil. Rev Bras Med Fam Comunidade. 2018;13(40):1-10. doi: https:// doi.org/10.5712/rbmfc13(40)1633

4. Quinn M, Babb P, Jones J, et al. Effect of screening on incidence of and mortality from cancer of cervix in England: evaluation based on routinely collected statistics. BMJ. 1999;318(7188):904-8. doi: https://doi. org/10.1136/bmj.318.7188.904

5. Instituto Nacional de Câncer José Alencar Gomes da Silva. Estimativa 2020: incidência de câncer no Brasil [Internet]. Rio de Janeiro: INCA; 2019 [acesso 2021 jun 17]. Disponível em: https:/www.inca.gov.br/sites/ ufu.sti.inca.local/files/media/document/estimativa-2020incidencia-de-cancer-no-brasil.pdf

6. Instituto Nacional de Câncer José Alencar Gomes da Silva. Atlas On-line de Mortalidade [Internet]. Rio de Janeiro: INCA; c1996-2014. Taxas de mortalidade por câncer, brutas e ajustadas por idade pelas populaçóes mundial e brasileira, por 100.000, segundo sexo, faixa etária, localidade e por período selecionado; [atualizado 2019 maio 30; acesso 2021 jun 17]. Disponível em: https://mortalidade.inca.gov.br/MortalidadeWeb/pages/ Modelo03/consultar.xhtml\#panelResultado

7. Girianelli VR, Gamarra CJ, Azevedo e Silva G. Os grandes contrastes na mortalidade por câncer do colo uterino e de mama no Brasil. Rev Saúde Pública.
2014;48(3):459-67. doi: https://doi.org/10.1590/ S0034-8910.2014048005214

8. Azevedo e Silva G, Jardim BC, Ferreira VM, et al. Mortalidade por câncer nas capitais e no interior do Brasil: uma análise de quatro décadas. Rev Saúde Pública. 2020;54:126. doi: https://doi.org/10.11606/s15188787.2020054002255

9. Instituto Nacional de Câncer José Alencar Gomes da Silva. Monitoramento das açóes de controle dos cânceres do colo do útero e de mama. Informativo Detecção Precoce [Internet]. 2019;10(2):1-7 [acesso 2021 jun 17]. Disponível em: https://www.inca.gov.br/publicacoes/ informativos/informativo-deteccao-precoce-no-2-2019

10. Nayar R, Wilbur DC, editors. The bethesda system for reporting cervical cytology: definitions, criteria, and explanatory notes. 3rd ed. New York: Springer; 2015.

11. Instituto Nacional de Câncer José Alencar Gomes da Silva. Manual de gestão da qualidade para laboratório de citopatologia [Internet]. 2. ed. rev. ampl. Rio de Janeiro: INCA; 2016 [acesso 2021 jun 17]. Disponível em: https://www.inca.gov.br/sites/ufu.sti.inca.local/ files//media/document//livro_completo_manual_ citopatologia-2016.pdf

12. Thuler LCS, Zardo LM, Zeferino LC. Perfil dos laboratórios de citopatologia do Sistema Único de Saúde. J Bras Patol Med Lab. 2007;43(2):103-14. doi: https:// doi.org/10.1590/S1676-24442007000200006

13. Stofler MECW, Nunes RD, Rojas PFB, et al. Avaliação do desempenho da citologia e colposcopia comparados com a histopatologia no rastreamento e diagnóstico das lesôes do colo uterino. ACM Arq Catarin Med. 2011;40(3):30-6.

14. Instituto Nacional de Câncer José Alencar Gomes da Silva. Diretrizes brasileiras para o rastreamento do câncer do colo do útero [Internet]. 2. ed. rev. atual. Rio de Janeiro: INCA; 2016 [acesso 2021 jun 17]. Disponível em: https:/www.inca.gov.br/sites/ ufu.sti.inca.local/files//media/document//diretrizes paraorastreamentodocancerdocolodoutero_2016_ corrigido.pdf

15. Tavassoli FA, Devilee P, editors. Cancer pathology and genetics: pathology and genetics of tumours of the breast and female genital organs. 3rd ed. Lyon: International Agency for Research on Cancer; 2003. Chapter 5, Tumours of the uterine cervix; p. 259-289.

16. Instituto Nacional de Câncer, Coordenação de Prevenção e Vigilância. Falando sobre câncer do colo do útero [Internet]. Rio de Janeiro: INCA; 2002 [acesso 2021 jun 17]. Disponível em: https://bvsms. saude.gov.br/bvs/publicacoes/inca/falando_cancer_ colo_utero.pdf

17. Instituto Nacional de Câncer José Alencar Gomes da Silva. Sistema de informação do câncer: manual 
preliminar para apoio à implantação. Rio de Janeiro: INCA; 2013 [acesso 2021 jun 17]. Disponível em: https://www.inca.gov.br/sites/ufu.sti.inca.local/ files//media/document//sistema-informacao-cancermanual.pdf

18. R Core Team. Version 4.1.0. [software]. 2021 May 18 [cited 2021 junho 17]. Available from: http:// www.R-project.org/

19. von Karsa L, Suonio E, Lignini T, et al., editors. Current status and future directions of breast and cervical cancer prevention and early detection in Belarus. France: International Agency for Research on Cancer; 2012 (Working group report; vol. 6)

20. Tomazelli JG, Migowski A, Ribeiro CM, et al. Avaliação das açóes de detecção precoce do câncer de mama no Brasil por meio de indicadores de processo: estudo descritivo com dados do Sismama, 2010-2011. Epidemiol Serv Saúde. 2017;26(1). doi: https://doi. org/10.5123/S1679-49742017000100007

21. Joshi C, Kujur P, Thakur N. Correlation of Pap smear and colposcopy in relation to histopathological findings in detection of premalignant lesions of cervix in a tertiary care centre. Int J Sci Stud [Internet]. 2015 [cited 2021 junho 17];3(8):55-60. Available from: http://www.ijss-sn.com/uploads/2/0/1/5/20153321/ ijss_nov_oa13.pdf

22. Dayal S. Clinico-histological analysis of non-neoplastic lesions of cervix. J Pathol Nep. 2018;8(1):1276-9. doi: https://doi.org/10.3126/jpn.v8i1.19453

23. Bousquat A, Giovanella L, Fausto MCR, et al. A atenção primária em regióes de saúde: política, estrutura e organização. Cad Saúde Pública. 2019;35(Suppl. 2):e00099118. doi: https://doi.org/10.1590/0102$311 \mathrm{X} 00099118$

24. Brito-Silva K, Bezerra AFB, Chaves LDP, et al. Integralidade no cuidado ao câncer do colo do útero: avaliação do acesso. Rev Saúde Pública. 2014;48(2):240-8. doi: https://doi.org/10.1590/ S0034-8910.2014048004852

25. Farias ACB, Barbieri AR. Seguimento do câncer de colo de útero: estudo da continuidade da assistência à paciente em uma região de saúde. Esc Anna Nery. 2016;20(4):e20160096. doi: https://doi. org/10.5935/1414-8145.20160096 